This document is the accepted manuscript version of the following article:

Densing, M. (2020). The value of flexible selling: power production with storage for spinning reserve

provision. European Journal of Operational Research, 281(1), 141-151. https://doi.org/10.1016/

j.ejor.2019.08.012

This manuscript version is made available under the CC-BY-NC-ND 4.0 1icense http://creativecommons.org/ 1icenses/by-nc-nd/4.0/

\title{
The value of flexible selling: Power production with storage for spinning reserve provision
}

\author{
M. Densing*
}

Energy Economics Group, Paul Scherrer Institute, 5232 Villigen PSI, Switzerland

\begin{abstract}
A stored commodity is sold under a capacity constraint depending on an exogenous random market price, and, alternatively, a service contract can be provided in which the selling amount must be held constant over time independent of price changes. The seller of the commodity is assumed to optimize the trade-off between the received payment for the provision of the service and the loss of flexibility by the reduced selling on the market. The chosen setup allows for closed-form solutions, such that the analysis is of theoretical interest. A potential application is hydropower storage optimization against exogenous electricity prices with the option to enter contracts for providing spinning reserve; spinning reserve is needed to stabilize largescale power systems. A single-period model is considered, and the storage level of the commodity is bounded from below in expectation. These simplifications allow a closed-form solution of bang-bang type, even under our assumption of an infinite probability space.
\end{abstract}

Keywords: OR in energy, stochastic programming, duality theory, hydropower optimization, spinning reserve

\footnotetext{
*tel.: +4156 3102598; fax: +41563104411

Email address: martin.densing@psi.ch (M. Densing)
} 


\section{Introduction}

The problem is to choose optimally either to sell a stored commodity at a market price, where the price is exogenously given and uncertain over time, or to provide a paid service that locks the selling amount on the market to a constant rate over time. The potential application is stored hydropower optimization, where the commodity is water stored in a reservoir and the constant operation is needed to provide the service of spinning reserve. The considered optimization model assumes some abstractions to allow for analytical insight. Hence the current state of analysis is foremost of mathematical interest, but the optimization problem is formulated generally such that it may be also useful for applications, for example as a benchmark. The research questions are as follows.

- Under which conditions should a commodity seller having access to a limited amount of commodity lock-in into constant selling?

- Can we formulate the conditions in closed-form for a non-trivial model?

Formulated in more concrete terms of power production, which we mainly will use in the remaining part of the paper, we seek an optimal dispatch decision, that is, how much a seller should produce over time for different markets, which are in our case the energy and the spinning reserve market. The seller is assumed to be a price-taker.

Explicit optimal solutions of dispatch of several stochastic stored-energy production problems are given in Densing (2013), where the storage level is assumed to be bound in expectation, which is a weaker constraint than a bound in every scenario (i.e. for each realization of the random variables). We continue with a similar model for spinning reserve, motivated also by the 
suggestions for future research of Weitzel \& Glock (2018) in their review of energy storage systems models, where they propose as first recommendation to follow up.

Numerical stochastic power production models for combined spot and reserve markets are considered in various papers. Combined bidding in both markets is considered in Ni et al. (2004), where the constraints on hydropower storage levels are in expectation (as in our case), and in Swider (2007), but without storage constraints. Wu et al. (2008) considers a riskconstrained hydrothermal scheduling algorithm including a reserve market. Boomsma et al. (2014) and Abgottspon et al. (2014), consider combined bidding using a multi-stage stochastic program with risk-aversion. Sioshansi (2015) considers a day-ahead two-stage storage optimization with several reservoirs. Campos et al. (2016) provides a detailed model for combined bidding rules, where - contrary to our model - the storage value of hydropower is given exogenously. As an example of technical details, Chazarra et al. (2016) consider in their stochastic programming model the non-constant relation between power and change in storage level (head-effect), and also the flow times between storage reservoirs.

What is missing in the above modeling is analytical insight. Our modeling of a - relatively stylized - spinning reserve provision allows for an exact solution. This solution provides for example an analytical (and not only numerical) condition for the switch between free production and the lock-in for a service provision. Although the model is relatively abstract, the solution is already non-trivial; for example, the switching condition is necessary but not sufficient under some parameter settings, and it is explicitly formulated in terms of the mean absolute deviation of the random price (variability), whereas without a closed-form solution, such functional dependences are 
concealed and can only be partially retrieved through numerical sensitivity runs. Moreover, a stochastic model is necessary, because the price variability determines the solution, which can be shown explicitly by the closed-form solution. Hence, our model provides some basic analytical insight in the value of lost flexibility, and therefore helps in the understanding of pricing of flexibility (options) in energy markets. It is also one of the few, non-trivial stochastic optimization problems for which a closed-form solution is known to the knowledge of the author.

To allow for an explicit solution of a stochastic optimization problem, the setting must be restrictive: Apart from the constraint on storage level in expectation, we assume for example a single storage reservoir over a single time step, only a lower bound on storage level, and a continuous distribution function of price. Nevertheless, the formulation is extensible to multiple time steps or discontinuous distribution function, but we would not be able to categorize the optimal solution; such extensions are treated in a follow-up publication.

The paper is organized as follows. In Sec. 2 the modeling assumptions are presented, and subsequently, the assumptions are discussed in view of the hydropower application with optional spinning reserve provision; a purely theoretically oriented reader may skip this subsection. In Sec. 3, the primal optimization problems is stated. The relevant properties of the dual problem are in Sec. 4. The closed-form solution of the primal problem is given in Sec. 5. In Sec. 6, the necessary condition for spinning reserve is compared with historical prices for spinning reserve in Switzerland, and we provide an illustrative example how the model could be used in principle. Eventually, we conclude. 


\section{Modeling assumptions}

Spinning reserve is needed to counterbalance unforeseen power fluctuations between energy supply and demand in an electricity grid. To provide spinning reserve, flexible production capacity is sold to the transmission system operator (TSO); the exact amount of production from this capacity is no longer under the seller's control because the system operator can request up- and down-regulation of energy production for balancing energy, whereas the seller has to fix production over time at a so-called set-point to be able to follow the requests; the fixed production at the set-point can still be sold on the energy market. A seller who provides spinning reserve loses flexibility on the market, because she cannot ramp-up the selling to full capacity when prices are high (in case up-regulation is contracted) or ramp-down selling when prices are low (in case of down-regulation). On the other hand, the seller receives an upfront payment for the provision, and separately, there is usually a reimbursement for the provided balancing energy. The maximal amount of ramp-up and ramp-down power capacity is agreed in the contract with the system operator. We assume that both ramp-up and ramp-down capacity must be provided and that the seller must provide capacity symmetrically, that is, power must be able to be maximally regulated down by $-u_{a}$ and maximally regulated up by the same amount $u_{a}$ (MW; the unithere: Megawatt, for illustrative purpose only — depends how the time period of the model is chosen in particular instances). If spinning reserve is provided, the maximal down-regulation $-u_{a}$ implies that the fixed production must be at least $u_{a}$ (without the requested balancing energy), and the maximal up-regulation of $u_{a}$ implies that $2 u_{a} \leq u_{\max }$, where $u_{\max }$ is the total capacity of the seller. 
In summary, we make the following assumptions.

- The seller provides capacity $\pm u_{a}(\mathrm{MW})$ over a certain time period to the system operator. The time period is larger than the time unit of market price change.

- The seller sells over this time period the fixed amount $u_{a}$ per time unit at the market price $S$, which is varying over the time units and therefore modeled as a random variable.

- The system operator pays the seller $p_{a} u_{a}(\mathrm{EUR} / \mathrm{MW} \cdot \mathrm{MW})$ for providing the service.

- The requested downward balancing energy equals the upward balancing energy over the time period

- The reimbursements for the actually requested balancing energy are not considered. Alternatively, we assume that these reimbursements are included in the payment $p_{a}$ of the provision.

- We consider only selling of stored energy and no pumping operation. Non-linearities between the conversion from stored energy into power are neglected, for example head-effects of stored hydropower or partload efficiency-losses. The technical lower bound of production is assumed to be zero, such that the capacity interval for reserve power can be chosen to be $\left[0,2 u_{a}\right]$, with the so-called set-point $u_{a}$ (of production at a constant rate) in the middle (Fig. 1, left).

The assumptions enable analytical insight in the theoretical problem of flexible selling at a random market price with the alternative of a provision to lock-in (partially) into a constant selling rate. The considered modeling 
may also be considered from an aggregated system view, where the seller comprises, for example, of all stored hydropower plants.

\subsection{Discussion of model assumptions}

For application-oriented readers, the assumptions are motivated in a series of remarks.

Remark 1 (Balancing services). In Europe, balancing services for (active) power system stability are usually categorized by the maximally allowed ramp-up (and ramp-down) time: primary reserve (control) power has to be fully available very quickly after an imbalance (several seconds); secondary reserve power, which has to be fully available later on (several minutes); and tertiary reserve, which starts afterwards. Primary and secondary service providers must be usually on-line (spinning), because there is no time for start-up (because of rotational inertia). We focus on secondary (spinning) reserve.

The requested balancing energy of spinning reserve for the total system in a given time unit is usually proportionally distributed over the participating producers according to contract size (in terms of maximal up- and downregulation). We also assume proportionality in this paper.

Remark 2 (Idiosyncrasy of balancing power services). Currently implemented reserve market schemes of spinning reserve are diverse; see for example the different provision schemes for various European countries (Ocker et al., 2016, Tab. I), which do not yet include the separate reimbursement schemes for balancing energy. Moreover, reserve market schemes are constantly adapted to the increasingly liberalized markets and changes in supply and demand structures. This is also a reason why we focus on basic characteristics, which is mainly the lock-in of production, to avoid idiosyncrasies which 


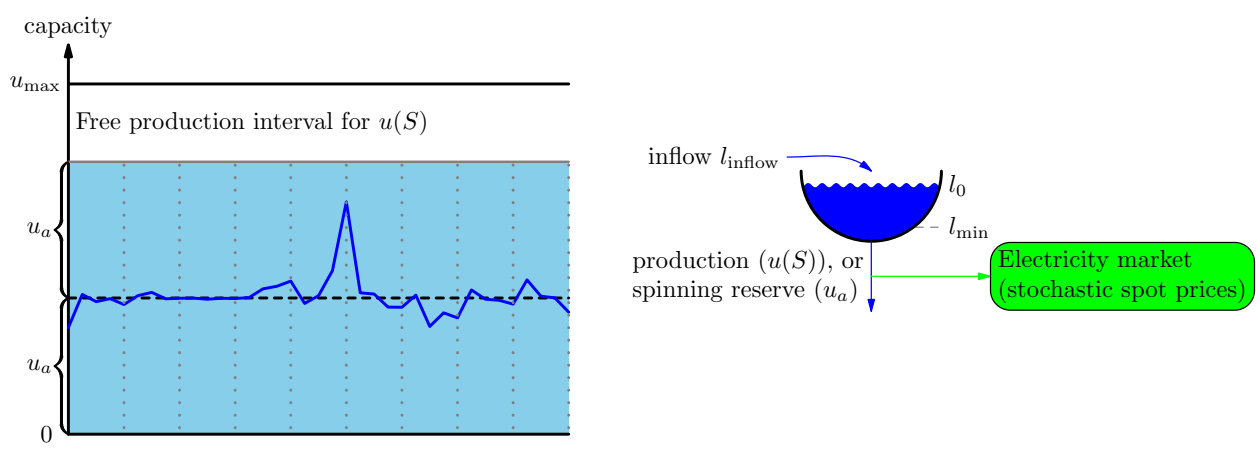

Figure 1: Left: Example of balancing power of secondary spinning reserve. The illustrative curve is the requested balancing power in total for Switzerland $(1.11 .2016,13 \mathrm{~h}-22 \mathrm{~h})$, which correspond to a scaling of $u_{a}=400 \mathrm{MW}$ (Swissgrid, 2017); Right: Model setup

are even changing over time.

Remark 3 (Symmetry of up- and down regulation). The assumption that the maximal possible up-regulation equals the required down-regulation in a contract is called symmetry (Ocker et al., 2016), and motivated by two reasons.

The first reason is practical: The requirement of symmetric up- and down regulation currently holds for contracts for example for the Swiss market and therefore also on a system level (Ocker et al., 2016; Swissgrid, 2017). As an illustration, Fig. 1 (left) shows vertically the range of size $2 u_{a}$ (color: light blue) of required capacity reservation for symmetric balancing; the remaining range up to maximum capacity can be used for (free) production that is allowed to depend on a realization of a sufficiently high market price $S$, which varies over the considered time period; the sold energy on the market is $u_{a}$ (const.) $+u(S)$. The obligation in Switzerland for symmetry may be removed in the future to allow more inflexible contracting (e.g. only for down-regulation).

The second reason is conceptual: Short-term imbalances in an electricity 
system are caused by superposition of many independent causes (e.g. demand variations in grid nodes, hourly production schedule switches), such that we can assume by the central limit theorem a normal distribution for the deviations. The forecast can be considered to be the mean of the distribution. Hence, from a system's perspective, to cover most of the imbalance up to a sufficiently small probability, the required amount of up-regulation equals the down-regulation by the symmetry of the probability density function. By the foregoing assumption of proportionality, this holds also for a single contract.

Remark 4 (Netting of up- and down balancing energy). The assumption that over the time horizon the sum of requested down-regulation energy equals the sum of up-regulation energy is motivated by the following two reasons; nevertheless, this strong assumption must be validated case-by-case.

The first reason is that if the required down-regulation energy (in total for the system) would consistently differ from the up-regulation energy over a sufficiently long time horizon, then the system operator would adjust the forecast of power and remove this offset. In other words, any systematic, observable offset in required balancing energy can be removed by the system operator by scaling the production schedules.

The second reason is empirical. In Switzerland, the time period of the service is currently weekly with time steps $t, t+1, \ldots$ of 15 minutes. We consider for each week the sum of requested secondary balancing energy for the system, netted over up-and down-regulation energy: $\sum_{t=1}^{T} u_{t}+d_{t}$, $T:=4 \cdot 168$. For example, in Switzerland in the year 2016, the required (system) set-point is $400 \mathrm{MW}$ for upward and downward regulation for the whole system (Swissgrid, 2017). Hence, the balancing energy in week $w$ as a 
fraction of fixed production at this set-point is $f_{w}:=\left|\sum_{t=1}^{T} u_{t}+d_{t}\right| /(400 T)$. We get $f_{w}<6 \%$ for all weeks $w$, and the average is $1.5 \%$ over the weeks in year 2016. Hence, the cumulative net balancing energy is small compared with the cumulative constant rate production at the set-point. Hence, the former is the more relevant for cumulative production amounts and for emptying the energy storage.

Remark 5 (Reimbursements for balancing energy). Reimbursement rules for balancing energy are idiosyncratic for different countries and changing over time. The current rules for Switzerland are as follows (Swissgrid, 2017).

- The required balancing energy for the whole system at a specific time step is proportionally attributed to the requested regulations of the single contracts. The time-horizon $T$ of the contract is a week.

- The seller receives from the system operator $u_{a} B_{t}^{+} \alpha^{+} S_{t}$ (EUR) for up-regulation of energy in time unit $t$, where $u_{a}$ is the contract size, $B_{t}^{+} \in[0,1]$ is the requested regulation, $\alpha^{+}:=1.2, S_{t}$ is the market (spot) price of energy, and $S_{t}$ and $B_{t}^{+}$are random variables.

- The seller pays to the system operator $u_{a} B_{t}^{-} \alpha^{-} S_{t}$ (EUR) for downregulation of energy, where $\alpha^{-}=0.8$, and $B_{t}^{-}$is a random variable.

Hence, the expected reimbursement over a week is

$$
\begin{aligned}
& \mathbb{E}\left[u_{a} \sum_{t=1}^{T}\left(\alpha^{+} B_{t}^{+} S_{t}-\alpha^{-} B_{t}^{-} S_{t}\right)\right] \\
& \stackrel{(*)}{=} u_{a} \sum_{t=1}^{T}\left(\alpha^{+} \mathbb{E}\left[B_{t}^{+}\right]-\alpha^{-} \mathbb{E}\left[B_{t}^{-}\right]\right) \mathbb{E}\left[S_{t}\right] \\
& \stackrel{(* *)}{=} u_{a} \sum_{t=1}^{T}\left(\alpha^{+}-\alpha^{-}\right) \mathbb{E}\left[B_{t}^{+}\right] \mathbb{E}\left[S_{t}\right]
\end{aligned}
$$


where in $(*)$ we assume that the (random) energy price $S_{t}$ is stochastically independent of the (random) balancing energy $B_{t}^{ \pm}$for all $t$, and in (**) we assume that the up- and down regulation energy levels-out in expectation for all $t: \mathbb{E}\left[B_{t}^{+}\right]=\mathbb{E}\left[B_{t}^{-}\right]$(a precise definition of $\mathbb{E}[\cdot]$ is in the model formulation below). Because the balancing energy can be assumed to be time-independent in expectation, $b:=\mathbb{E}\left[B_{t}^{+}\right]$, the expected payment (1) simplifies to $u_{a} \tilde{p}_{a}$, where $\tilde{p}_{a}:=b\left(\alpha^{+}-\alpha^{-}\right) \sum_{t=1}^{T} \mathbb{E}\left[S_{t}\right]$. Hence, under the above assumptions, the reimbursement for the balancing energy $\tilde{p}_{a}$ could be added to the provision payment $p_{a}$.

Alternatively, the payment for balancing energy can be neglected altogether in the modeling because - at least for Switzerland - the reimbursement for balancing energy is small compared with the provision payment (see Sec. 6). Indeed, in Switzerland in year 2016, the absolute value of balancing energy at a point in time is on average only $9 \%$ of the required maximum up- and down-regulation. Hence, net-payments are small compared to the required locked-in production capacity.

Note that the previous time steps $t, t+1, \ldots$ have a length of 15 minutes, and the time scale of $S_{t}$ is accordingly. By contrast, in this particular setting, the random variable $S$ of the model assumption (p. 6) represents the distribution of the spot prices over the entire week.

Remark 6 (Non-linearities). We exclude non-linearities, such as head-effects of stored hydropower, which is the non-linearity between storage level and produced energy. The size of the head-effect depends on the quotient of the usable range of energy level in the storage and of the pen-stock height down to the turbine. We assume that the time step is relatively small (e.g. a week or shorter) and that the pen-stock is relatively high, such that changes in 
storage levels can be neglected.

\section{Single-period model with spinning reserve provision}

The optimal control problem is to maximize expected profit by selling on the free market and by entering the service. We consider a single-period model where the lower bound on the storage level has to be fulfilled in expectation (Fig. 1, right). The expectation of a random variable $X$ is denoted by $\mathbb{E}[X]$, which is in mathematical terms an integration over a probability space $(\Omega, \mathcal{F}, \mathbb{P}): \mathbb{E}[X]=\int X \mathrm{~d} \mathbb{P}$, which is $\int_{-\infty}^{\infty} x f(x) d x$ in case $X$ has a probability density function $f(x)$. We assume that the spot market price $S$ is a random variable having a continuous distribution function, and that $S$ is integrable and non-negative $\left(S \in L_{+}^{1}(\Omega ; \mathbb{R})\right)$. The control function of free selling depends on the market price, that is, $u: \mathbb{R}_{+} \rightarrow \mathbb{R}_{+}$, and $u(S(\omega))$ is the sold energy (per time unit) in a scenario $\omega \in \Omega$. We use also the notation $U=u(S)$, where $U$ is a random variable, implying that $u$ is a Borel-measurable function.

For the (mathematical) model formulation, the unit of time is chosen to be the time-period of the model (e.g. a day or week); with this choice, energy (by power produced) has always the same value as the power. Hence, capacity and energy do not require conversion factors and can be related in equations directly, which facilitates notation. The translation from other units is for example as follows. If (original) capacities $\tilde{u}(S)$ (and $\tilde{u}_{a}$ etc.) have MW as unit, and the time period of modeling is 1 week, then the capacities in the mathematical formulation are $u(S)=168 \tilde{u}(S)$, with the stored energy amount in unit MWh is for the weekly operation.

The maximal capacity and the usable storage (level) are assumed to be 
related by $u_{\max }>l>0$, where the usable storage is $l=l_{0}+l_{\text {inflow }}-l_{\text {min }}$ with initial level $l_{0}$, expected inflow $l_{\text {inflow }}$, and minimal level $l_{\text {min }}$. Hence, we assume that it is not allowed to sell with full capacity $u_{\max }$; in other words, the storage is a scarce resource. By entering the service contract of amount $u_{a}$, the symmetrical interval of capacity around the set-point $u_{a}$ must be reserved: $\left[0,2 u_{a}\right] \cdot p_{a}$ is the payment for the service provision per unit of contract size. Under the assumptions stated in the introduction, the single-period optimization problem for expected profit with the ability to lock-in for spinning reserve provision is

$$
\left.\begin{array}{rlr}
\max _{u(\cdot), u_{a}} \mathbb{E}\left[S\left(u(S)+u_{a}\right)\right]+p_{a} u_{a} & \\
\mathbb{E}\left[u(S)+u_{a}\right] \leq l, & (q \geq 0) \\
u(S)+2 u_{a} \leq u_{\max }, & (Z \geq 0) \\
u(S), u_{a} \geq 0, &
\end{array}\right\}
$$

with variable $u_{a} \in \mathbb{R}_{+}$and the foregoing control-function $u$. Constraints on random variables are always meant to be fulfilled in every scenario (i.e. almost surely (a.s.), which will be mentioned sometimes explicitly for clarity). Hence, the second constraint in (P) reads more explicitly as $u(S(\omega))+2 u_{a} \leq$ $u_{\text {max }}$ for almost every $\omega \in \Omega$.

Problem (P) is feasible under the assumptions made (e.g. $u(S) \equiv 0, u_{a}=$ $0)$. We may interpret the single-period model $(\mathrm{P})$ as a model that is applied over several time periods with a rolling (single-period) horizon, or, alternatively, as a steady-state model as in Densing (2013). The model (P) is a two-stage stochastic linear problem (Kall \& Mayer, 2011): The first-stage (here-and-now) decision is $u_{a}$, and the second-stage decision is $U=u(S)$, which depends on the scenario $\omega: U(\omega)=u(S(\omega))$. Note that the second stage represents in applications usually a time period (e.g. day or week), and 
not a single point in time. The Lagrange-multiplier of the storage constraint is $q \in \mathbb{R}_{+}$, and the Lagrange-multiplier of the upper capacity constraint is $Z \geq 0$ (a.s.), which is a random variable; or, written pointwise, $Z(\omega) \geq 0$ for almost every $\omega \in \Omega$.

The case without spinning reserve, that is, by setting $u_{a}=0$ in Problem (P), is studied in Densing (2013), where an optimal (bang-bang) solution is given: $\hat{u}(S)=u_{\max } 1_{\{S \geq \hat{q}\}}$, where $\hat{q}$ is a quantile of the spot price given by $\mathbb{P}[S \geq \hat{q}]=l / u_{\max }$, and where $1_{A}$ denotes the indicator function of an event $A \in \mathcal{F}$, and $\mathbb{P}[A]$ the probability of an event $A$. The optimal solution was derived by using the more accessible dual problem (Densing, 2013),

$$
\min _{q \geq 0}\left(q l+u_{\max } \mathbb{E}\left[(S-q)^{+}\right]\right)
$$

where $(\cdot)^{+}=\max (\cdot, 0)$. Hence, we investigate the dual problem in our case, too.

\section{Dual problem}

As we will show in this section the dual problem of $(\mathrm{P})$ can be solved analytically, leading to a closed form solution of (P) eventually, presented in Sec. 5. The dual problem of $(\mathrm{P})$ is

$$
\min _{q \geq 0} d(q)=\min _{q \geq 0}\left(q l+u_{\max }\left(\mathbb{E}\left[(S-q)^{+}\right]+\frac{1}{2}\left(p_{a}-\mathbb{E}[|S-q|]\right)^{+}\right)\right),
$$

where $(\cdot)^{+}=\max (\cdot, 0)$. The objective function of (D) is that of problem (2) (i.e. without spinning reserve) with an additional term of $1 / 2\left(p_{a}-\mathbb{E}[\mid S-\right.$ $q \mid])^{+}$. We will show formally that (D) is the dual of $(\mathrm{P})$ in the subsequent Lem. 2.

Remarkably, in the primal problem $(\mathrm{P})$, the spinning reserve provision is intermingled with the free market, whereas in the dual problem (D) the 
provision is a separate, additive term. The well-known interpretation applies: The primal problem $(\mathrm{P})$ is a resource allocation, whereas $(\mathrm{D})$ is a resource valuation. Indeed, the additive terms in (D) are valued with the dual price $q$, which gives a value of the usable storage, $q l$, a value of free market selling, $u_{\max } \mathbb{E}\left[(S-q)^{+}\right]$, and a value of spinning reserve provision, $\frac{1}{2}\left(p_{a}-\mathbb{E}[|S-q|]\right)^{+}$.

Let us investigate the dual objective function $d(q)$ in $(\mathrm{D})$ in more depth. The function contains the term $\mathbb{E}[|S-q|]$ (which is due to the symmetry assumption of the spinning reserve provision as we will shortly see). The median $m$, which is defined by $\mathbb{P}[S \leq m]=\frac{1}{2}$ for the continuously distributed random variable $S$, is related to this term as follows.

Lemma 1 (median). The median of an integrable random variable $X$ with continuous distribution function is

$$
m=\operatorname{argmin}_{q \in \mathbb{R}} \mathbb{E}[|X-q|] .
$$

Proof. In case $X$ has a continuous distribution function, the function $\mathbb{E}[\mid X-$ $q \mid]=\mathbb{E}\left[(X-q)^{+}\right]-\mathbb{E}\left[(X-q)^{-}\right]$is differentiable with respect to $q: \frac{\partial}{\partial q} \mathbb{E}[(X-$ $\left.q)^{+}\right]=-\mathbb{P}[X \geq q]$ and $\frac{\partial}{\partial q} \mathbb{E}\left[(X-q)^{-}\right]=\mathbb{P}[X \leq q]$, where $(\cdot)^{-}:=\min (\cdot, 0)$ and $(\cdot)^{+}:=\max (\cdot, 0)$ (see e.g. Rockafellar \& Uryasev (2002)).

In our case, $m>0$, because we assume that $S$ has a continuous distribution function and that $S \geq 0$. Consider now the last term in (D), which corresponds to the valuation of the spinning reserve provision. It follows from (3) that if the payment is relatively low, $p_{a} \leq \mathbb{E}[|S-m|]$, the term is zero for all $q$ (cf. Densing (2013)). Hence, the provision does not increase the objective dual value. 
Other structural properties of the dual problem (D) are given in the following lemma.

Lemma 2. (i) The objective function d can be rearranged to

$$
d(q)=q l+u_{\max } \max \left(\frac{1}{2}\left(\mathbb{E}[S]+p_{a}-q\right), \mathbb{E}\left[(S-q)^{+}\right]\right) .
$$

(ii) (Weak duality) Problem (D) is the dual of (P). The objective function value of $(\mathrm{P})$ is lower or equal to that of $(\mathrm{D})$ for any feasible solutions of $(\mathrm{P})$ and $(\mathrm{D})$.

(iii) The function $d$ is convex, with $\lim _{q \rightarrow \infty} d^{\prime}(q)=l$, and

$$
\lim _{q \searrow 0} d^{\prime}(q)=l-\frac{1}{2} u_{\max } \quad \text { if } p_{a}>\mathbb{E}[S] .
$$

Proof. (i): The second term of (4) is equivalent to the corresponding term in (D) because

$$
\begin{aligned}
& \max \left(\frac{1}{2}\left(\mathbb{E}[S-q]+p_{a}\right), \mathbb{E}\left[(S-q)^{+}\right]\right) \\
& =\max \left(\frac{1}{2}\left(\mathbb{E}\left[(S-q)^{+}\right]+\mathbb{E}\left[(S-q)^{-}\right]+p_{a}\right), \mathbb{E}\left[(S-q)^{+}\right]\right) \\
& =\mathbb{E}\left[(S-q)^{+}\right]+\max \left(\frac{1}{2}\left(-\mathbb{E}[|S-q|]+p_{a}\right), 0\right)
\end{aligned}
$$

where $(\cdot)^{-}=\min (\cdot, 0)$.

(ii) : The Lagrangian function $L$ of $(\mathrm{P})$ with Lagrange multipliers $q$ and $Z$ (as indicated in $(\mathrm{P})$ ) and with abbreviation $U=u(S)$ is $L\left(U, u_{a}\right)=\mathbb{E}[U(S-q-Z)]+u_{\max } \mathbb{E}[Z]+u_{a}\left(\mathbb{E}[S]+p_{a}-q-2 \mathbb{E}[Z]\right)+q l$

Note that the Lagrangian is built with an implicit summation by the ex- 
pected value (Pflug \& Ruszczyński, 2005, p. 165).

$$
\begin{aligned}
\sup _{U \geq 0, u_{a} \geq 0} L<\infty & \Longleftrightarrow\left\{\begin{array}{l}
S-q-Z \leq 0 \quad \text { a.s. } \\
\mathbb{E}[S]+p_{a}-q-2 \mathbb{E}[Z] \leq 0
\end{array}\right. \\
& \Longleftrightarrow\left\{\begin{array}{l}
Z \geq S-q \quad \text { a.s., } \\
\mathbb{E}[Z] \geq \frac{1}{2}\left(\mathbb{E}[S]+p_{a}-q\right) .
\end{array}\right.
\end{aligned}
$$

The dual function is

$$
\theta(q, Z):=\max _{\substack{\text { s.t. }(6), U \geq 0, u_{a} \geq 0}} L=q l+u_{\max } \mathbb{E}[Z]
$$

Hence, the dual problem is $\min \theta(q, Z)$ s.t. (6):

$$
\left.\begin{array}{rl}
\min _{Z, q} q l & +u_{\max } \mathbb{E}[Z] \\
Z & \geq S-q, \\
\mathbb{E}[Z] & \geq \frac{1}{2}\left(\mathbb{E}[S]+p_{a}-q\right), \\
Z & \geq 0, q \geq 0 .
\end{array}\right\}
$$

The random variable $Z$ enters the objective value function of (7) only as an expected value. Hence, the first constraint in (7) can be relaxed to $\mathbb{E}[Z] \geq \mathbb{E}[S]-q$, and the condition $Z \geq 0$ (a.s.) to $\mathbb{E}[Z] \geq 0$ ('a.s.' means in every scenario (almost surely)). The relaxed problem equals $\min _{q \geq 0} d(q)$, where $d(q)$ is given by (4). Hence, (D) is the dual problem, which implies in principle weak duality. We show weak duality also directly as follows. Let $U=u(S)$ and $u_{a}$ be a feasible solution of the primal problem (P). The 
objective function of the primal problem is

$$
\begin{aligned}
& \mathbb{E}[U S]+u_{a}\left(\mathbb{E}[S]+p_{a}\right) \\
& =\mathbb{E}[U(S-q)]+q \mathbb{E}[U]+q u_{a}+u_{a}\left(\mathbb{E}[S-q]+p_{a}\right) \\
& \stackrel{(*)}{\leq} \mathbb{E}\left[\left(u_{\max }-2 u_{a}\right)(S-q)\right]+q l+u_{a}\left(\mathbb{E}[S-q]+p_{a}\right) \\
& \stackrel{(* *)}{\leq}\left(u_{\max }-2 u_{a}\right) \mathbb{E}\left[(S-q)^{+}\right]+q l+u_{a}\left(\mathbb{E}[S-q]+p_{a}\right) \\
& =u_{\max } \mathbb{E}\left[(S-q)^{+}\right]+q l+u_{a}\left(\mathbb{E}[S-q]-2 \mathbb{E}\left[(S-q)^{+}\right]+p_{a}\right) \\
& \stackrel{(* * *)}{=} u_{\max } \mathbb{E}\left[(S-q)^{+}\right]+q l+u_{a}\left(-\mathbb{E}\left[(S-q)^{-}\right]-\mathbb{E}\left[(S-q)^{+}\right]+p_{a}\right) \\
& =u_{\max } \mathbb{E}\left[(S-q)^{+}\right]+q l+u_{a}\left(p_{a}-\mathbb{E}[|S-q|]\right) \\
& \leq u_{\max } \mathbb{E}\left[(S-q)^{+}\right]+q l+\frac{1}{2} u_{\max }\left(p_{a}-\mathbb{E}[|S-q|]\right)^{+},
\end{aligned}
$$

where for $(*)$ the upper bound on feasible controls is used: $U+2 u_{a} \leq u_{\max }$, and the feasibility in energy level: $\mathbb{E}[U]+u_{a} \leq l$; for $(* *)$ the upper bound together with the non-negativity of feasible controls is used: $u_{\max }-2 u_{a} \geq 0$; in $(* * *)$ a split is used: $\mathbb{E}[S-q]=\mathbb{E}\left[(S-q)^{+}\right]+\mathbb{E}\left[(S-q)^{-}\right],(\cdot)^{-}:=\min (\cdot, 0)$; and the last inequality uses again the feasibility of control: $u_{a} \leq \frac{1}{2} u_{\max }$. The last term is the objective function of the dual problem (D).

$(i v)$ : The function (4) is according to $(i i)$ a maximum of two convex functions; $\mathbb{E}\left[(S-q)^{+}\right]$is convex because $(S(\omega)-q)^{+}$is convex in $q$ for all $\omega \in \Omega$, and because integration is monotone.

In case $q \notin\left\{\tilde{q}: p_{a}=\mathbb{E}[|S-\tilde{q}|]\right\}$, the derivative of the function (4) is

$$
d^{\prime}(q)=l-\frac{1}{2} u_{\max } 1_{\left\{p_{a}>E[|S-q|]\right\}}-u_{\max } \mathbb{P}[S \geq q] 1_{\left\{p_{a}<E[|S-q|]\right\}} .
$$

where $\frac{\partial}{\partial q} \mathbb{E}\left[(S-q)^{+}\right]=-\mathbb{P}[S \geq q]$ is used, which holds in case $S$ has a continuous distribution function (Rockafellar \& Uryasev, 2002).

By Lem. 2 (iii), $d$ is convex and therefore continuous. Also by (iii), $\lim _{q \rightarrow \infty} d^{\prime}(q)=l$, which implies (because $l>0$ by assumption) that there 
exists a sufficiently large $M>0$ such that $d(q)$ is strictly increasing for $q \geq M$. Hence, the infimum of the continuous function $d$ on $[0, \infty)$ is attained on the compact subset $[0, M]$. Therefore, the dual problem (D) attains its minimum.

The characterization of the minimizers of (D) are given in the following lemma, where we need the term $\mathbb{E}[|S-m|]$, which is the mean absolute deviation (MAD) from the median $m$ of the price $S$. Note that in other literature the MAD is more commonly defined not with respect to $m$, but to the mean of $S$.

Lemma 3 (Minimizer of dual problem). Let $\hat{q}$ be an optimal solution of the dual problem $(\mathrm{D}): \min _{q \geq 0} d(q)$.

(i) Assume $p_{a} \leq \mathbb{E}[|S-m|]$. Then $d$ is differentiable, and $\hat{q}$ is given by

$$
u_{\max } \mathbb{P}[S \geq \hat{q}]=l,
$$

which implies $\hat{q}>0$.

(ii) Assume $p_{a}>\mathbb{E}[|S-m|]$. Then d has two (non-differentiable) kinkpoints $\left\{q_{l}, q_{r}\right\}=\left\{q: p_{a}=\mathbb{E}[|S-q|]\right\}$, where $q_{l} \leq m \leq q_{r}$ for the left and right kink-point. The optimal solution is

$$
\hat{q}= \begin{cases}0 & \text { if } l \geq \frac{1}{2} u_{\max } \wedge p_{a}>\mathbb{E}[S], \\ q_{l} & \text { if } u_{\max } \mathbb{P}\left[S \geq q_{l}\right] \geq l \geq \frac{1}{2} u_{\max }, \\ q_{r} & \text { if } u_{\max } \mathbb{P}\left[S \geq q_{r}\right] \leq l \leq \frac{1}{2} u_{\max }, \\ q & \text { if } u_{\max } \mathbb{P}\left[S \geq q_{l}\right]<l \wedge \frac{1}{2} u_{\max } \leq l, \\ q & \text { if } u_{\max } \mathbb{P}\left[S \geq q_{r}\right]>l \wedge \frac{1}{2} u_{\max } \geq l,\end{cases}
$$


Table 1: Categorization of the optimal dual solution $\hat{q}$ according to Lem. 3

\begin{tabular}{|c|c|c|c|}
\cline { 2 - 4 } \multicolumn{1}{c|}{} & $\hat{q}=q_{l}$ & $\hat{q}=q_{r}$ & $\hat{q} \notin\left\{q_{l}, q_{r}\right\}$ \\
\hline$\hat{q}>0$ & $(10 \mathrm{~b})$ & $(10 \mathrm{c})$ & $(9),(10 \mathrm{~d}),(10 \mathrm{e}): u_{\max } \mathbb{P}[S \geq \hat{q}]=l$ \\
\hline$\hat{q}=0$ & - & - & $(10 \mathrm{a})$ \\
\hline
\end{tabular}

where $q$ is given by $u_{\max } \mathbb{P}[S \geq q]=l$, which implies $q>0$ (as in $(i)$ ), and $\wedge$ denotes the logical 'and'.

Proof. (i): In case $p_{a} \leq \mathbb{E}[|S-m|]$ the function (D) simplifies to $d(q)=$ $l q+u_{\max } \mathbb{E}\left[(S-q)^{+}\right]$, which has stationary condition $l-u_{\max } \mathbb{P}[S \geq q]=0$. Because we generally assume that $l<u_{\max }$, we have $\mathbb{P}[S \geq \hat{q}]<1$, which implies $\hat{q}>0$ by the general assumption $S \geq 0$.

(ii): The function (4) is the maximum of two differentiable functions. Kink-points can occur where the functions are equal: $\frac{1}{2}\left(\mathbb{E}[S-q]+p_{a}\right)=$ $\mathbb{E}\left[(S-q)^{+}\right]$, which is $p_{a}=\mathbb{E}[|S-q|] . f(q)=\mathbb{E}[|S-q|]$ is a convex function with minimum at the median $m$ by Lem. 1 ; hence $p_{a}=f(q)$ has at most two solutions: $q_{l} \leq m \leq q_{r}$.

At $q=0$, and if $p_{a}>\mathbb{E}[|S|]$, the derivative (8) is non-negative, i.e. $d^{\prime}(0) \geq 0$ if and only if $l \geq \frac{1}{2} u_{\max }$. In this case, $p_{a}>\mathbb{E}[|S|]=\mathbb{E}[S] \Longrightarrow$ $p_{a}>\mathbb{E}[|S-m|]$, and $q_{l} \leq 0$. In the other case, $p_{a}<\mathbb{E}[|S|], d^{\prime}(0) \geq 0$ implies $u_{\max } \leq 0$, which is excluded by assumption.

The kink-point $q_{l}$ is a minimizer if the left-derivative is non-positive and the right-derivative is non-negative at $q_{l}$, which is according to (8) if and only if $l-\frac{1}{2} u_{\max } \geq 0$ and $l-u_{\max } \mathbb{P}\left[S \geq q_{l}\right] \leq 0$, where the second inequality implies $q_{l}>0$. For $\hat{q}=q_{r}$ the inverse inequalities hold.

The case $p_{a} \leq \mathbb{E}[|S-m|]$ (Lem. 3, (9)) corresponds to the problem without spinning reserve (Densing, 2013). All the cases of Lem. 3 are categorized 
in Tab. 1 and depicted in Fig. 2, where the graph of $d$ is drawn with and without the additive term of spinning reserve provision in the reformulation (4). For the examples in the figure, we assume a normal distribution of the price (see Sec. 6.3 for the numerical calculation of expected values). If the usable storage amount $l$ is high, that is, $l \geq \frac{1}{2} u_{\max }$, then the reserve provision can decrease the price of the storage constraint, but the service still increases the optimal expected profit (Case (10a)). If the usable storage amount $l$ is sufficiently low, then the price of the storage constraint is usually increased (Case (10b) and (10c)). Clearly, if the payment for the reserve provision is low, then the dual problem reduces to pure free market selling, and the additive term of the reserve provision is zero (Case (9)).

Two extreme cases are shown in Fig. 3. If the storage amount is extremely scarce, then entering spinning reserve provision is not profitable even for relatively high provision payments (Case (10e)). It is more profitable to sell the precious storage in the (short) time periods of very high prices. If the storage amount is extremely abundant, then the reserve provision is also not profitable even for relatively high provision payments (Case (10d)). Indeed, with plenty of storage, selling at the free market is almost constant over time and nearly at full capacity, which is similar to the constant selling rate for spinning reserve provision, such that the lock-in into the provision gives not a higher profit if it is merely moderately paid. In other words, the option of reserve provision in Case (10e) and (10d) does not (additively) contribute to the optimal dual objective value. Hence, the necessary condition that $p_{a}>\mathbb{E}[|S-m|]$ is not always sufficient for the reserve provision to be profitable. 
Case (10b):

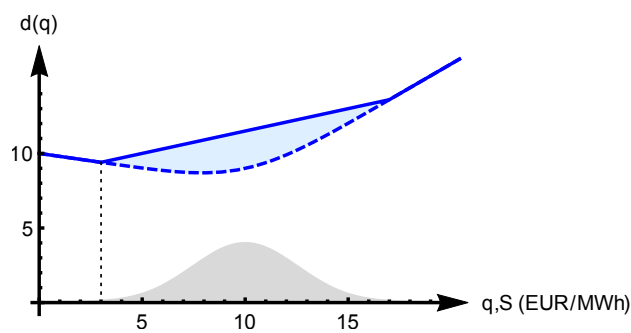

Case (10c):

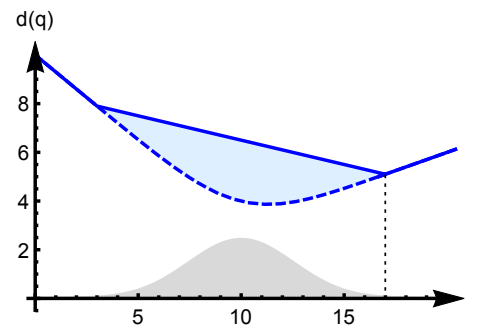

Case (10b) and (10c):

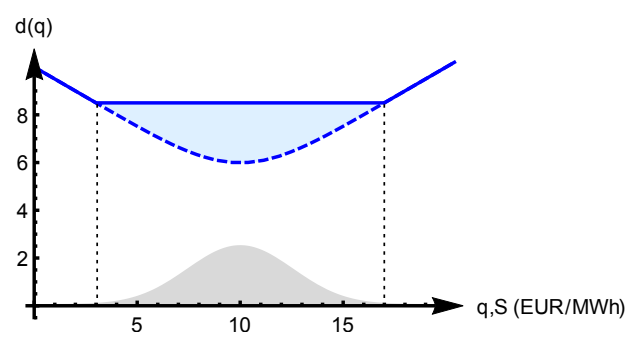

Case (10a):

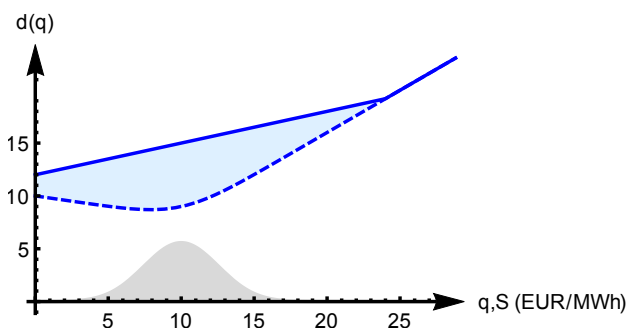

Case (10c):

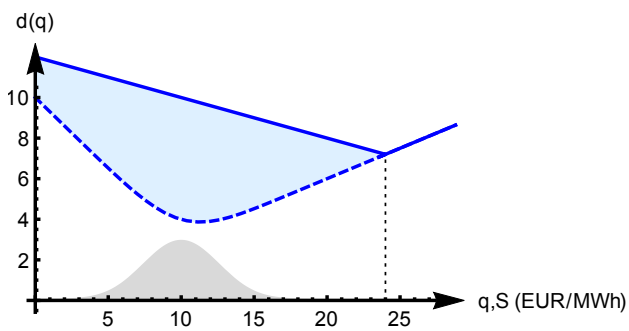

Case (9):

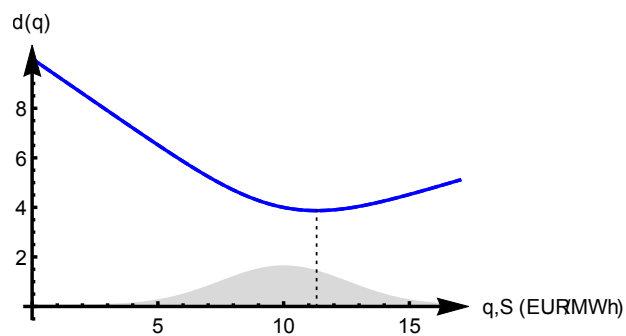

Figure 2: Solid line (-): Dual function $d: \mathbb{R}^{+} \rightarrow \mathbb{R}^{+}$. Dotted line (---): $d$ with term of spinning reserve provision subtracted. Dotted vertical lines: Minimizer of $d$. Probability density of random variable $S \sim N(10, \sigma=2.5)$ in gray. Parameters: $u_{\max }=1$; payment $p_{a}$ of reserve provision is assumed to be profitable: $p_{a}>\mathbb{E}[|S-m|]$ (except Case (9)). Top: High storage level $(l=0.8): u_{\max } \mathbb{P}\left[S \geq q_{l}\right] \geq l \geq \frac{1}{2} u_{\max }$. Case (10b) with $p_{a}=7$, and Case (10a) with $p_{a}=14$. Mid: Low storage level $(l=0.3): u_{\max } \mathbb{P}\left[S \geq q_{r}\right] \leq l \leq \frac{1}{2} u_{\max }$ (Case (10c)). Mid-left and right: $p_{a}=7$ and $p_{a}=14$, respectively. Bottom-left: Special case $l=\frac{1}{2} u_{\max }(l=0.5)$, corresponding to Case (10b) and (10c). Bottom-right: Low provision payment: $p_{a}=1, l=0.3$ (Case (9)). 

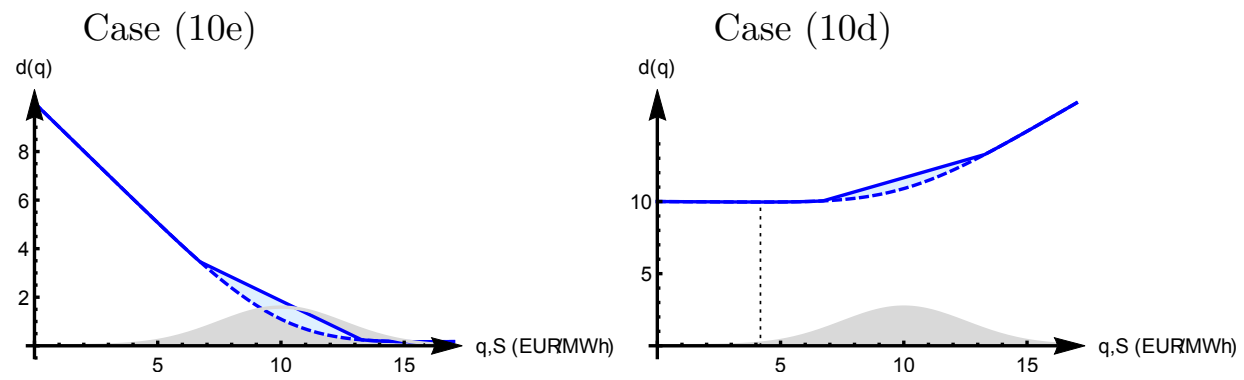

Figure 3: Dual function $d$ (solid line) in case where necessary condition for reserve provision is fulfilled but payment for provision still too low: $p_{a}=3.5>\mathbb{E}[|S-m|] \approx 2$. Dotted line (---): Dual function with term of reserve provision subtracted. Left: Very low storage level $(l=0.01): l<u_{\max } \min \left(\frac{1}{2}, \mathbb{P}\left[S \geq q_{r}\right]\right)$; Right: Very high storage level $(l=0.99)$ : $l>u_{\max } \max \left(\frac{1}{2}, \mathbb{P}\left[S \geq q_{l}\right]\right)$. Parameters: $u_{\max }=1$; random variable $S \sim N(10, \sigma=2.5)$

\section{Optimal solution of primal problem}

The foregoing necessary condition for a profitable reserve provision is also restated in the following proposition, where as a main result an optimal primal solution is given.

Proposition 1. Let $\hat{q}$ be any optimal solution of the dual problem (D).

(i) (Strong Duality). The primal and dual problem (P) and (D) have the same optimal objective value.

(ii) (Case of active storage constraint: $\hat{q}>0$ ). If $\hat{q}>0$, then an optimal solution of problem $(\mathrm{P})$ is

$$
\left.\begin{array}{rl}
\hat{u}(S) & =\left(u_{\max }-2 \hat{u}_{a}\right) 1_{\{S \geq \hat{q}\}} \\
\hat{u}_{a} & =\frac{1}{2} u_{\max }-\frac{l-\frac{1}{2} u_{\max }}{1-2 \mathbb{P}[S \leq \hat{q}]}
\end{array}\right\}
$$

(iii) (Case of non-active storage constraint: $\hat{q}=0$ ). If $\hat{q}=0$, then an 
optimal solution of problem $(\mathrm{P})$ is

$$
\left.\begin{array}{rlr}
\hat{u}(S) & =0 \quad \text { a.s. } \\
\hat{u}_{a} & =\frac{1}{2} u_{\max }
\end{array}\right\}
$$

where a.s. means in every scenario (almost surely). $\hat{q}=0$ if and only if $p_{a} \geq \mathbb{E}[S]$ and $\frac{1}{2} u_{\max } \leq l$.

(vi) (Necessary condition for spinning reserve provision). If an optimal solution of problem $(\mathrm{P})$ has a non-zero spinning reserve provision $\hat{u}_{a}$, then the payment for the provision must be higher than the mean absolute deviation (MAD) from the median $m$ of the price $S$, that is, $\hat{u}_{a}>0 \Longrightarrow p_{a}>\mathbb{E}[|S-m|]$, where $m$ is given by $\mathbb{P}[S \leq m]=\frac{1}{2}$.

For the proof, we need a result for the special case where the optimal solution $\hat{q}$ in (D) is a quantile (cf. Lem. 3 (iii)); as mentioned, this case corresponds to the pure free market selling problem as in Densing (2013).

Lemma 4. Assume $\hat{q}$ is the quantile given by $u_{\max } \mathbb{P}[S \geq \hat{q}]=l$. Then $\hat{q}>0$, and in (11) we obtain $\hat{u}_{a}=0$.

Proof. Because we generally assume $l<u_{\max }$, we have $\mathbb{P}[S \geq \hat{q}]<1$, which implies $\hat{q}>0$ by the general assumption $S \geq 0$. By inserting $u_{\max } \mathbb{P}[S \geq$ $\hat{q}]=l$ into the optimal solution $\hat{u}_{a}$ in (11) we obtain

$$
\hat{u}_{a}=\frac{1}{2} u_{\max }-\frac{u_{\max } \mathbb{P}[S \geq \hat{q}]-\frac{1}{2} u_{\max }}{1-2 \mathbb{P}[S \leq \hat{q}]}=0,
$$

where we used $\mathbb{P}[S \geq q]+\mathbb{P}[S \leq q]=1$ for any $q(\mathbb{P}[S=q]=0$ because $S$ has a continuous distribution function by general assumption).

Proof. (Prop. 1) Assume that (i) in Prop. 1 holds (strong duality). Then (iv) follows from the additivity of the term of reserve provision in the dual opti- 
mization problem (D): If $p_{a} \leq \mathbb{E}[|S-m|]$, then this term is zero (cf. remark after Lem. 1).

We prove statement (i), (ii), and (iii) for each of the cases of dual optimal solution $\hat{q}$ of Lem. 3 as listed in Tab. 1. In each case, we show that the proposed primal optimal solutions are feasible (dual feasibility holds trivially: $\hat{q} \geq 0$ ); and that the problems are strongly dual, that is, problem (D) has the same optimal objective value as the primal problem $(\mathrm{P})$. Indeed, we know that problems (P) and (D) are weakly dual for feasible solutions by Lem. 2 .

(1) Proposed optimal solution is feasible in problem (P): (1.a) Feasible constraint on storage.

Case $\hat{q}>0$. The constraint on storage is fulfilled because

$$
\begin{aligned}
\mathbb{E}\left[\hat{U}+\hat{u}_{a}\right] & \stackrel{(*)}{=}\left(u_{\max }-2 \hat{u}_{a}\right) \mathbb{P}[S \geq \hat{q}]+\hat{u}_{a} \\
& \stackrel{(* *)}{=}\left(u_{\max }-2\left(\frac{1}{2} u_{\max }+\frac{l-\frac{1}{2} u_{\max }}{2 \mathbb{P}[S \leq \hat{q}]-1}\right)\right) \mathbb{P}[S \geq \hat{q}] \\
& \quad+\frac{1}{2} u_{\max }+\frac{l-\frac{1}{2} u_{\max }}{2 \mathbb{P}[S \leq \hat{q}]-1} \\
& =l,
\end{aligned}
$$

where for $(*)$ the optimal solution $\hat{U}$ of $(11)$ is substituted, and for $(* *)$ the optimal solution $\hat{u}_{a}$ of (11). The last equation follows directly.

Case $\hat{q}=0$. By Lem. 3 it holds that $p_{a} \geq \mathbb{E}[S]$ and $\frac{1}{2} u_{\max } \leq l$. Because in case $\hat{q}=0$ the proposed optimal solution has also $\hat{U}=0$ according to Prop. 1 (iii), it follows $\mathbb{E}\left[\hat{U}+\hat{u}_{a}\right]=\frac{1}{2} u_{\max } \leq l$.

(1.b) Feasible upper and lower bounds of capacity.

Case $\hat{q}>0$ and $\hat{q} \in\left\{q_{l}, q_{r}\right\}$ ( $\hat{q}$ is kink-point). In case $\hat{q}$ is a kink-point of the 
dual function (D) and $\hat{q}>0$, the production is

$$
\begin{aligned}
\hat{U}+2 \hat{u}_{a} & \stackrel{(*)}{=}\left(u_{\max }-2 \hat{u}_{a}\right) 1_{\{S \geq q\}}+2 \hat{u}_{a} \\
& \stackrel{(* *)}{=} u_{\max }+\frac{2 l-u_{\max }}{2 \mathbb{P}[S \leq \hat{q}]-1} 1_{\{S \geq q\}} \stackrel{(* * *)}{\leq} u_{\max } .
\end{aligned}
$$

where for $(*)$ the optimal solution $\hat{U}$ of (11) is substituted (as in (14)); for $(* *)$ the optimal solution $\hat{u}_{a}$ is substituted (and terms are rearranged); and $(* * *)$ follows in case of a left kink-point $\left(q_{l}=\hat{q}\right.$; Case (10b)) because the nominator in the fraction is $2 l-u_{\max } \geq 0$, and the denominator is $2 \mathbb{P}\left[S \leq q_{l}\right]-1 \leq 0$ (because the left kink-point is lower or equal to the median of $S$ ), which yields in combination a non-positive fraction in (15). In case of a right-kink point $\left(q_{r}=\hat{q}\right.$; Case (10c)), we apply for $(* * *)$ the same reasoning with reserved signs.

The proposed optimal solution (11) for $\hat{u}_{a}$ fulfills $\hat{u}_{a} \leq \frac{1}{2} u_{\max }$ as follows. In case of a left kink-point, both nominator and denominator of $\hat{u}_{a}$ in (11) are non-positive, whereas in case of a right kink-point, both are non-negative; hence, $\hat{u}_{a} \leq \frac{1}{2} u_{\max }$. This implies for solution (11) that $\hat{U} \geq 0$. The nonnegativity of $\hat{u}_{a}$ follows from

$$
\hat{u}_{a}=\frac{1}{2} u_{\max }-\frac{l-\frac{1}{2} u_{\max }}{1-2 \mathbb{P}[S \leq \hat{q}]}=\frac{l-u_{\max } \mathbb{P}[S \geq \hat{q}]}{1-2 \mathbb{P}[S \geq \hat{q}]} \geq 0
$$

where the inequality follows from the conditions in Case (10b) and (10c).

Case $\hat{q}>0$ and $\hat{q} \notin\left\{q_{l}, q_{r}\right\}$. In this case, $u_{\max } \mathbb{P}[S \geq \hat{q}]=l$ (see Tab. 1 ). Hence, $\hat{u}_{a}=0$ by Lem. 4 , which ensures the lower bound on capacity. For the upper bound, we have $\hat{U}+2 \hat{u}_{a}=\hat{U}=u_{\max } 1_{\{S \geq q\}} \leq u_{\max }$.

Case $\hat{q}=0$. The proposed optimal solution is $\hat{u}=\frac{1}{2} u_{\max }$ and $\hat{U}=0$ according to Prop. 1 (iii), which is obviously feasible.

(2) Strong duality.

In the following, for each case of dual minimizer $\hat{q}$, we start with the pri- 
mal optimal objective function and show equality to the dual. Note that according to Lem. 3 (see also Figs. 2 and 3), the minimal value of the dual optimization problem (D) is always attained at a point $\hat{q}$ that corresponds to a value of the dual problem without the spinning reserve position, except in case $\hat{q}=0$.

Case $\hat{q}>0$ and $\hat{q} \in\left\{q_{l}, q_{r}\right\}$. Let $\hat{u}_{a}$ be an optimal primal solution (11) and $\hat{q}$ an optimal solution of the dual problem in case where $\hat{q}$ is a kink-point. The optimal objective value of problem $(\mathrm{P})$ is

$$
\begin{aligned}
& \mathbb{E}\left[\left(u_{\max }-2 \hat{u}_{a}\right) S 1_{\{S \geq \hat{q}\}}+\hat{u}_{a} S\right]+\hat{u}_{a} p_{a} \\
& =u_{\max } \mathbb{E}\left[S 1_{\{S \geq \hat{q}\}}\right]+\hat{u}_{a}\left(\mathbb{E}[S]-2 \mathbb{E}\left[S 1_{\{S \geq \hat{q}\}}\right]+p_{a}\right) \\
& =u_{\max } \mathbb{E}\left[S 1_{\{S \geq \hat{q}\}}\right]+\hat{u}_{a}\left(\mathbb{E}\left[S 1_{\{S \leq \hat{q}\}}\right]-\mathbb{E}\left[S 1_{\{S \geq \hat{q}\}}\right]+p_{a}\right) \\
& =u_{\max } \mathbb{E}\left[S 1_{\{S \geq \hat{q}\}}\right]+\hat{u}_{a}\left(-\mathbb{E}\left[(S-\hat{q})^{-}\right]+\hat{q} \mathbb{P}[S \leq \hat{q}]\right. \\
& \left.\qquad-\mathbb{E}\left[(S-\hat{q})^{+}\right]-\hat{q} \mathbb{P}[S \geq \hat{q}]+p_{a}\right) \\
& =u_{\max } \mathbb{E}\left[S 1_{\{S \geq \hat{q}\}}\right]+\hat{u}_{a}\left(p_{a}-\mathbb{E}[|S-\hat{q}|]+\hat{q}(\mathbb{P}[S \leq \hat{q}]-\mathbb{P}[S \geq \hat{q}])\right) \\
& \stackrel{(*)}{=} u_{\max } \mathbb{E}\left[S 1_{\{S \geq \hat{q}\}}\right]+\hat{u}_{a} \hat{q}(2 \mathbb{P}[S \leq \hat{q}]-1) \\
& \stackrel{(* *)}{=} u_{\max } \mathbb{E}\left[S 1_{\{S \geq \hat{q}\}}\right]+\frac{u_{\max }(\mathbb{P}[S \leq q]-1)+l}{2 \mathbb{P}[S \leq q]-1} \\
& =u_{\max } \mathbb{E}\left[S 1_{\{S \geq \hat{q}\}}\right]+u_{\max } \hat{q} \mathbb{P}[S \leq q]-u_{\max } \hat{q}+\hat{q} l \\
& =u_{\max } \mathbb{E}\left[(S-\hat{q}) 1_{\{S \geq \hat{q}\}}\right]+u_{\max } \hat{q}(\mathbb{P}[S \geq q]+\mathbb{P}[S \leq q])-u_{\max } \hat{q}+\hat{q} l \\
& =u_{\max } \mathbb{E}\left[(S-\hat{q})^{+}\right]+\hat{q} l \\
& =d(\hat{q}),
\end{aligned}
$$

where in $(*)$ the dual optimality was used in case of kink-points, which implies $p_{a}=\mathbb{E}[|S-\hat{q}|]$ (see Lem. 3 (ii)), and in (**) the optimal solution (11) for $\hat{u}_{a}$ is inserted. The last term is the objective function of the dual problem (D) with $p_{a}=\mathbb{E}[|S-\hat{q}|]$. 
Case $\hat{q}>0$ and $\hat{q} \notin\left\{q_{l}, q_{r}\right\}$. In this case, we have $u_{\max } \mathbb{P}[S \geq \hat{q}]=l$, which implies $\hat{u}_{a}=0$ by Lem. 4 . To show strong duality, we can simplify (17) by specializing to $\hat{u}_{a}=0$ :

$$
\begin{aligned}
& \mathbb{E}\left[\left(u_{\max }-2 \hat{u}_{a}\right) S 1_{\{S \geq \hat{q}\}}+\hat{u}_{a} S\right]+\hat{u}_{a} p_{a} \\
& =u_{\max } \mathbb{E}\left[S 1_{\{S \geq \hat{q}\}}\right] \\
& =u_{\max } \mathbb{E}\left[(S-\hat{q}) 1_{\{S \geq \hat{q}\}}\right]+u_{\max } \hat{q} \mathbb{P}[S \geq q] \\
& =u_{\max } \mathbb{E}\left[(S-\hat{q})^{+}\right]+\hat{q} l,
\end{aligned}
$$

which is the dual function $d(\hat{q})$ without the option of spinning reserve.

Case $\hat{q}=0$. It follows from Lem. 2 (iv) and Lem. 3 that $d(0)$ is the minimum of the dual function $d$ if and only if $p_{a}>\mathbb{E}[S]$ and $l \geq \frac{1}{2} u_{\max }$. Furthermore, if $\hat{q}=0$, the primal objective values is for the proposed solution of Prop. 1 (iv)

$$
\begin{aligned}
& \mathbb{E}\left[\left(u_{\max }-2 \hat{u}_{a}\right) S 1_{\{S \geq \hat{q}\}}+\hat{u}_{a} S\right]+\hat{u}_{a} p_{a} \\
& =\mathbb{E}\left[\left(u_{\max }-2 \hat{u}_{a}\right) S+\hat{u}_{a} S\right]+\hat{u}_{a} p_{a} \\
& =\frac{1}{2} u_{\max }\left(\mathbb{E}[S]+p_{a}\right),
\end{aligned}
$$

which is $d(0)$. Hence, strong duality holds also in this case.

Figure 4 shows the optimal spinning reserve provision $\hat{u}_{a}$ as a function of the provision payment $p_{a}$ in the example of a normally distributed price $S$. If condition (vi) of Prop. 1 is violated, that is, the payment for the service is lower than $\mathbb{E}[|S-m|]$ (i.e. mean absolute deviation of the price from its median; MAD), then entering the service is not optimal. The condition is independent of the storage level. Note that MAD is commonly defined with respect to the mean of $S$, whereas in our case it is the median. As mentioned, the median enters because of the symmetry of the spinning reserve provision.

Figure 4 shows also that the necessary condition is not sufficient for entering the service, and moreover two qualitatively different cases: If the 

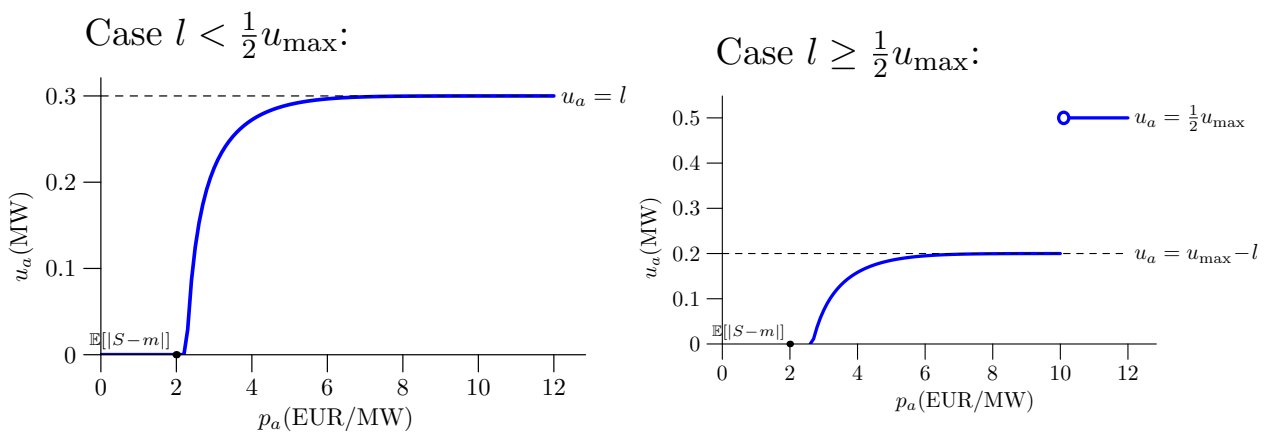

Figure 4: Spinning reserve provision $u_{a}$ as a function of the payment $p_{a}$ for provision. Parameters: $u_{\max }=1$; random variable $S \sim N(10, \sigma=2.5)$; Left: relatively low storage level $l=0.3$; Right: relatively high storage level $l=0.8$

stored amount $l$ is relatively scarce compared with the capacity fo selling, that is, $l<\frac{1}{2} u_{\max }$, then the spinning reserve provision $\hat{u}_{a}$ continuously increases to the maximal level allowed by the storage level, whereas if the stored amount is relatively abundant, $l \geq \frac{1}{2} u_{\max }$, then $\hat{u}_{a}$ also gradually phases in, but to a relatively lower level $u_{\max }-l$, which can be lower or higher than $l$ (e.g. a lower value is on the right of Fig. 4). In this case, $\hat{u}_{a}$ as a function of $p_{a}$ has a discontinuity at point $p_{a}=\mathbb{E}[S]$. It follows from Lem. 2 that in this case, if $p_{a} \nearrow \mathbb{E}[S]$, then $\hat{q} \searrow 0$, where $\hat{q}$ is the optimal solution of the dual problem. Hence, because the free market selling $\hat{u}(S)$ is a bang-bang solution at the maximal left-over capacity whenever $S \geq \hat{q}$ according to Prop. 1 (ii), free market selling is becoming more frequent when $p_{a} \nearrow \mathbb{E}[S]$, but the selling amounts are at a lower level of the left-over capacity. If $p_{a}$ is sufficiently high by surpassing the discontinuity point, then $\hat{q}=0$, which correspond to Case (10a) in Lem. 3 and to Fig. 2 (top-right).

In the special case $l=\frac{1}{2} u_{\max }$, the optimal reserve provision (11) yields always production at the maximally allowed set-point $\frac{1}{2} u_{\max }$, and there is no free market selling: $\hat{U}=0$. For the dual problem, this corresponds to 
Case (10b) combined with (10c) of Lem. 3 and to Fig. 2 (bottom-left).

Eventually, we check whether the objective value is continuous in case $l \geq \frac{1}{2} u_{\max }$. By considering the limits $p_{a} \nearrow \mathbb{E}[S]$ and $\hat{q} \searrow 0$, it can be directly checked that the objective value of problem $(\mathrm{P})$ reduces to $u_{\max } \mathbb{E}[S]$. By inserting the optimal solution for $p_{a} \geq \mathbb{E}[S]$, which is $\hat{u}_{a}=\frac{1}{2}$ and $\hat{U}=0$ according to Prop. 1 (iii), into the objective function, we get at point $p_{a}=$ $\mathbb{E}[S]$ the same value. Hence, the objective value is continuous with respect to $p_{a}$ at $p_{a}=\mathbb{E}[S]$.

\section{Empirical evidence and illustrative example}

\subsection{Historical prices for (secondary) spinning reserve provision}

As mentioned, contracting rules for system services in market areas over the world are heterogeneous and likely to change over time. We confine ourselves to compare historical prices with the obtained necessary condition for profitable spinning reserve provision. We consider results of the weekly auctions of secondary spinning reserve provision in Switzerland in the years 2016 and 2017. Taking Switzerland as an example is appropriate because the majority of domestic, flexible power production is hydropower, which correspond to our model (limited, stored commodity), and currently the contracting for reserve power is such that up- as well as down-regulation must be provided symmetrically, which also fits our model.

In Switzerland, the weekly auction of the service is currently a pay-asbid auction, that is, there is no single price (Swissgrid, 2017). We take the volume-weighted average price and scale it to an hourly price by dividing by $7 \cdot 24$. Note that this price is just for providing the capacity, but not yet for the balancing energy payments. Indeed, in the case of Switzerland, 


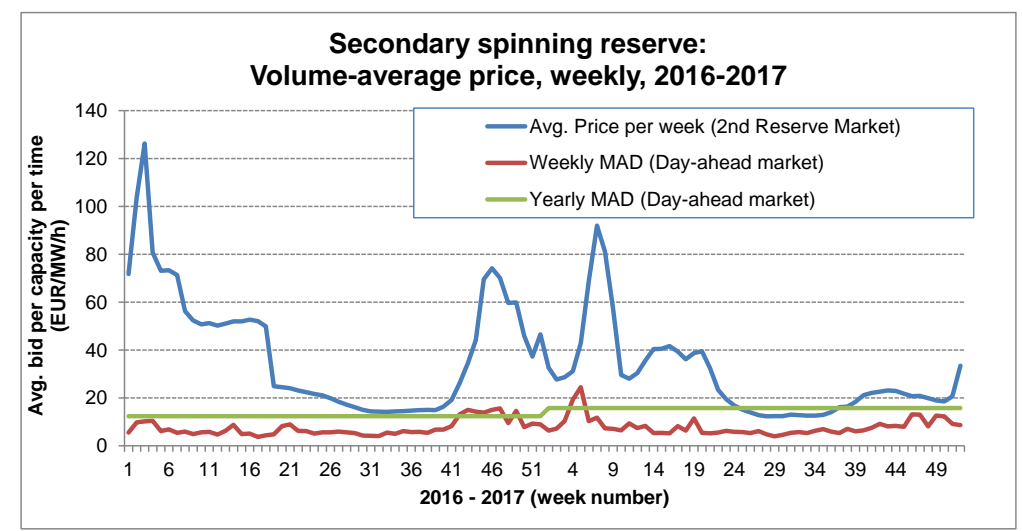

Figure 5: Historical prices of (secondary) spinning reserve provision in Switzerland, and mean absolute deviation (MAD) from the median of the hourly day-ahead electricity price

the energy payments are on average an order of magnitude smaller ${ }^{1}$. We compare the price with the mean absolute deviation from the median (MAD) of the hourly electricity price from the day-ahead market (EPEX, 2017). The comparison in Fig. 5 shows that in most of the weeks, the average price is above the yearly and weekly MAD, which is in agreement with the necessary condition of Prop. 1 (iv) for a profitable reserve provision.

\subsection{Illustrative example of possible application}

The presented single-period model is mainly for theoretical insight. Nevertheless, we provide an illustrative example for a possible application. We consider (approximately) the aggregated stored hydropower capacity with the corresponding aggregated range of required spinning reserve of Switzerland. The input to the model is also the distribution of the hourly day-ahead spot price, which is assumed to be normally distributed $S \sim N(\mu, \sigma)$, taken from historical data of year 2016 (month: November) (EPEX, 2017). The

\footnotetext{
${ }^{1} 1-2$ EUR/MWh in year 2017, Swissgrid (2017)
} 
Table 2: Input data of example, and model output

\begin{tabular}{|c|c|}
\hline & value \\
\hline$\mu$ (mean of price) & $61 \mathrm{EUR} / \mathrm{MWh}$ \\
$\sigma$ (std.dev. of price) & $17 \mathrm{EUR} / \mathrm{MWh}$ \\
$75 \%$ price percentile & $73.8 \mathrm{EUR} / \mathrm{MWh}$ \\
$\tilde{l}$ & $1351 \mathrm{GWh}$ \\
$u_{\max }$ & $7 \mathrm{GW}$ \\
$p_{a}$ & $17 \mathrm{EUR} / \mathrm{MW} / \mathrm{h}$ \\
\hline$\hat{u}_{a}$ & $453.1 \mathrm{MW}$ \\
$\hat{q}$ & $73.4 \mathrm{EUR} / \mathrm{MWh}$ \\
\hline \hline
\end{tabular}

available energy in a month, denoted by $\tilde{l}$, is assumed to be the historical production of stored hydropower in Nov. 2016 (SFOE, 2017); the level for a (scaled) hourly unit time scale is $l=\tilde{l} /(24 \cdot 30)$. As further input, we assume that the payment for the spinning reserve provision is $17 \mathrm{EUR} / \mathrm{MW} / \mathrm{h}$, which is in the lower range of Fig. 5. First, the optimal dual value $\hat{q}$ was calculated (cf. Sec. 6.3 for implementational details); using $\hat{q}$, the optimal solution (11) yields the set-point of spinning reserve provision $\hat{u}_{a}$ and the remaining production term $\hat{u}(S)$. Input and result data are summarized in Tab. 2. In the chosen example, $\hat{q}$ is near the $75 \%$-quantile of the price, and the set-point $\hat{u}_{a}$ is slightly above the set-point demanded by the Swiss TSO (currently approx. $400 \mathrm{MW}$ ).

Figure 6 shows an illustration how the single-period model could be applied in principle for an aggregated view on Swiss stored hydropower. The data is for the first week in Nov. 2016. The free market production (11) is a bang-bang solution triggered by $\hat{q}$, and the lock-in production is at the 


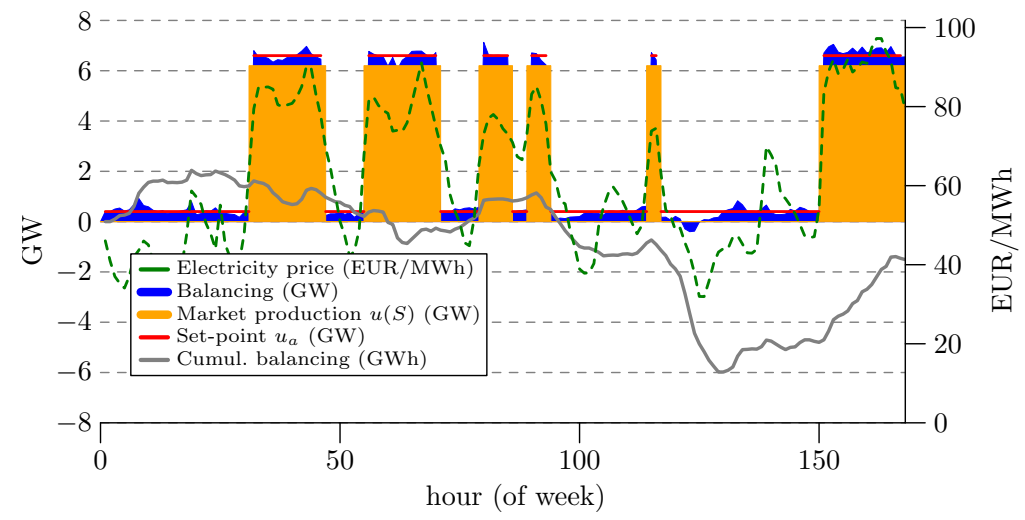

Figure 6: Illustration for use of model over time. Aggregated stored hydropower plants for Switzerland. Historical input data: Electricity price, balancing energy (and cumulative balancing energy) of one week of (secondary) spinning reserve provision (1.-7.Nov. 2016); set-point: $400 \mathrm{MW}$ (aggregated TSO's set-point); capacity: 7 GW. Resulting production threshold for free market selling: $73 \mathrm{EUR} / \mathrm{MWh}$

required set-point $\min \left(\hat{u}_{a}, 400 \mathrm{MW}\right)$. We note again that the constraint on the available energy has to hold in our model merely in expectation; for the considered sample path of random spot price, the constraint on the lower storage level is (slightly) violated, because the assumed available energy $\tilde{l}$ was assumed for an entire month, whereas production is already $438 \mathrm{GWh}$ for the specifically considered week. Hence, the model (in its current, simple form) would have to be updated for example daily to meet actual storage constraints (rolling horizon), and the usable storage level should be reduced by an empirical security margin.

\subsection{Implementational details}

The evaluation of the minimizer of the dual function $d(q)$ by using Lem. 3 involves the evaluation of $f(q):=\mathbb{E}[|S-q|]$. Alternatively, the dual (convex) function can be minimized directly, which involves the evaluation of $\mathbb{E}[(S-$ 
$q)^{+}$. For example, if we assume a normally distributed spot price $S \sim$ $N(\mu, \sigma)$ with mean $\mu$ and standard deviation $\sigma$, we get

$$
\begin{aligned}
\mathbb{E}\left[(S-q)^{ \pm}\right] & =\sigma \mathbb{E}\left[\left(X-\frac{q-\mu}{\sigma}\right)^{ \pm}\right], \\
\mathbb{E}\left[(X-q)^{+}\right] & =\frac{1}{\sqrt{2 \pi}} e^{-\frac{1}{2} q^{2}}+\frac{1}{2} q\left(\operatorname{erf}\left(\frac{q}{\sqrt{2 \pi}}\right)-1\right), \\
\mathbb{E}\left[(X-q)^{-}\right] & =-\mathbb{E}\left[(X-q)^{+}\right]-q, \\
\mathbb{E}[|X-q|] & =\mathbb{E}\left[(X-q)^{+}\right]-\mathbb{E}\left[(X-q)^{-}\right] \\
& =\sqrt{\frac{2}{\pi}} e^{-\frac{1}{2} q^{2}}+q \operatorname{erf}\left(\frac{q}{\sqrt{2}}\right),
\end{aligned}
$$

where $X \sim N(0,1)$ is the standard normal distribution, and $\operatorname{erf}(\cdot)$ is the error function. Note that the error function is not uniquely defined in literature and software; in our definition, $\operatorname{erf}\left(\frac{q}{\sqrt{2}}\right):=\frac{2}{\sqrt{2 \pi}} \int_{0}^{q} e^{-2 t^{2}} d t$, which is the convention for example in the software MATHEMATICA, whereas in the widely-used optimization software GAMS, the definition is $\operatorname{errorf}(q):=\frac{1}{2}+$ $\frac{1}{2} \operatorname{erf}\left(\frac{q}{\sqrt{2}}\right)$. Eqs. (19) were used for Figs. 2, 3, 4 and Tab. 2.

\section{Conclusion}

We considered a profit maximization problem of a stored commodity that can be sold at the free market or that can alternatively be sold at a constant rate for providing a service. Exact solutions are provided in case of a single-period stochastic optimization model and where a bound on the commodity storage is formulated in expectation. The closed-form solution is relatively simple but required to consider several cases because different parameter settings lead to qualitatively different dual functions.

A possible application (with suitable extensions) is hydropower with a storage reservoir and an option for spinning reserve provision, but the model structure is general to accommodate possibly other applications. Apart 
from the analytical insight from the exact solution, which can already help decision makers, the exactly solvable problem may be used inside larger, only numerically solvable optimization problems. We indicated also that the fundamental decision to enter the service provision is not in disagreement with historical data.

If we stay inside the general assumption of the storage level constraints to hold merely in expectation, some extensions are possible. For example, it is possible to consider an upper bound of the storage (and not only a lower bound), pumping (and not only production), and extend the model to multiple periods, which is addressed in a follow-up paper. The long-term goal is to find exact solutions in case the storage level constraints are to hold almost surely (i.e. in every scenario if the setting is finite).

On the other hand, electricity (and especially stored hydropower) dispatch optimization of real-world use-cases remains considerably challenging because more technical details should be considered, which usually lead to non-linear or mixed-integer formulations; in this work, we neglected for example minimum running constraints, head effects of hydropower storages, and part-load efficiency losses.

Acknowledgment. The work was financially supported by the Swiss Federal Office of Energy (research grant SI/501308) and the Swiss Competence Center of Energy Research - Supply of Electricity (SCCER-SoE).

\section{References}

Abgottspon, H., Njálsson, K., Bucher, M. A., \& Andersson, G. (2014). Risk-averse medium-term hydro optimization considering provision of spinning reserves. In International Conference on Proba- 
bilistic Methods Applied to Power Systems (PMAPS). Durham, UK. doi:10.1109/PMAPS.2014.6960657.

Boomsma, T. K., Juul, N., \& Fleten, S.-E. (2014). Bidding in sequential electricity markets: The nordic case. European Journal of Operational Research, 238, 797-809. doi:10.1016/j.ejor.2014.04.027.

Campos, F. A., Roque, A. M. S., Sánchez-Úbeda, E. F., \& González, J. P. (2016). Strategic bidding in secondary reserve markets. IEEE Transactions on Power Systems, 31, 2847-2856. doi:10.1109/tpwrs.2015.2453477.

Chazarra, M., García-González, J., Pérez-Díaz, J. I., \& Arteseros, M. (2016). Stochastic optimization model for the weekly scheduling of a hydropower system in day-ahead and secondary regulation reserve markets. Electric Power Systems Research, 130, 67-77. doi:10.1016/j.epsr.2015.08.014.

Densing, M. (2013). Dispatch planning using newsvendor dual problems and occupation times: Application to hydropower. European Journal of Operational Research, 228, 321-330. doi:10.1016/j.ejor.2013.01.033.

EPEX (2017). EPEX Spot market data. URL: https ://www . epexspot.com.

Kall, P., \& Mayer, J. (2011). Stochastic Linear Programming: Models, Theory, and Computation volume 156 of International Series in Operations Research $\&$ Management Science. (2nd ed.). Springer. doi:10.1007/9781-4419-7729-8.

Ni, E., Luh, P. B., \& Rourke, S. (2004). Optimal integrated generation bidding and scheduling with risk management under a deregulated power market. IEEE Transactions on Power Systems, 19, 600-609. doi:10.1109/TPWRS.2003.818695. 
Ocker, F., Braun, S., \& Will, C. (2016). Design of European balancing power markets. In 13th International Conference on the European Energy Market (EEM). IEEE. doi:10.1109/eem.2016.7521193.

Pflug, G. C., \& Ruszczyński, A. (2005). Measuring risk for income streams. Computational Optimization and Applications, 32, 161-178. doi:10.1007/s10589-005-2058-3.

Rockafellar, R. T., \& Uryasev, S. (2002). Conditional value-at-risk for general loss distributions. Journal of Banking \& Finance, 26, 1443-1471. doi:10.1016/S0378-4266(02)00271-6.

SFOE (2017). Swiss electricity statistics. URL: http://www.bfe.admin.ch Swiss Federal Office of Energy.

Sioshansi, R. (2015). Optimized offers for cascaded hydroelectric generators in a market with centralized dispatch. IEEE Transactions on Power Systems, 30, 773-783. doi:10.1109/tpwrs.2014.2332501.

Swider, D. J. (2007). Simultaneous bidding in day-ahead auctions for spot energy and power systems reserve. International Journal of Electrical Power \&6 Energy Systems, 29, 470-479. doi:10.1016/j.ijepes.2006.11.005.

Swissgrid (2017). swissgrid - ancillary services. tenders download. URL: https://www.swissgrid.ch.

Weitzel, T., \& Glock, C. H. (2018). Energy management for stationary electric energy storage systems: A systematic literature review. European Journal of Operational Research, 264, 582-606. doi:10.1016/j.ejor.2017.06.052. 
Wu, L., Shahidehpour, M., \& Li, Z. (2008). GENCO's risk-constrained hydrothermal scheduling. IEEE Transactions on Power Systems, 23, 18471858. doi:10.1109/tpwrs.2008.2004748. 